\title{
GCU
}

Glasgow Caledonian

University

University for the Common Good

\section{Factor structure and reliability of the parent-informant strengths and difficulties questionnaire in a Scottish preschool sample}

McAloney-Kocaman, Kareena; McPherson, Kerri

Published in:

Early Education and Development

DOI:

10.1080/10409289.2017.1228367

Publication date:

2017

Document Version

Author accepted manuscript

Link to publication in ResearchOnline

Citation for published version (Harvard):

McAloney-Kocaman, K \& McPherson, K 2017, 'Factor structure and reliability of the parent-informant strengths and difficulties questionnaire in a Scottish preschool sample', Early Education and Development, vol. 28, no. 3, pp. 368-376. https://doi.org/10.1080/10409289.2017.1228367

\section{General rights}

Copyright and moral rights for the publications made accessible in the public portal are retained by the authors and/or other copyright owners and it is a condition of accessing publications that users recognise and abide by the legal requirements associated with these rights.

Take down policy

If you believe that this document breaches copyright please view our takedown policy at https://edshare.gcu.ac.uk/id/eprint/5179 for details

of how to contact us. 
Factor structure of the Parent Informant Strengths and Difficulties Questionnaire (SDQ) in a Scottish sample. 
Funding: None

Conflict of interest: The authors report no conflict of interest.

Ethical approval: Ethical approval was not sought for this research as it is a secondary analysis, however, the primary research was subject to ethical review. 


\begin{abstract}
Despite being a widely accepted measure of social, emotional and behavioural problems among children and young people, the factor structure underlying the Strengths and Difficulties Questionnaire (SDQ) is unclear. Recent research has suggested a need to consider a method bias in the SDQ as a separate latent construct. Using data from the 2006/2007 Growing Up in Scotland study, confirmatory factor analyses was performed with 2,500 parental SDQ reports of their four year old children, estimating and comparing three competing factor models - five factor, three factor, and six factor (method factor). Results indicated that the 6 factor solution which included an uncorrelated method factor was the best fitting model. Several items from the pro-social factor and peer problems factor loaded more highly on the method factor than the original factors, suggesting bias influences across the SDQ domains. As the SDQ is widely used internationally further research is required to investigate the consistency of the method factor within the SDQ structure and implications for use and interpretation of the measure.
\end{abstract}

Key words: SDQ: children; confirmatory factor analysis; Scotland 
In recent years there has been growing recognition that children and young people are at risk of mental health problems and that early onset is associated with increased lifetime risk (Murphy et al, 2012). This has led to a proliferation in the development and implementation of early interventions to prevent and treat mental health problems and promote social, emotional and behavioural wellbeing in children and young people, and an associated requirement for psychometrically sound screening and outcomes assessment tools. While a range of assessment tools are available to both clinicians and researchers, one of the most frequently used is the Strengths and Difficulties Questionnaire (SDQ) (Goodman, 1997).

The SDQ has been promoted over alternative instruments because it assesses both positive and negative aspects of development and interpersonal relationships. In its original form, four difficulties sub-scales measure conduct problems, emotional problems, hyperactivity and peer problems and a strengths sub-scale measures prosocial behaviour (Goodman, 1997). The SDQ is free to use and with 25 items it is shorter than many of the alternatives, which increases its utility as a screening tool (Stone et al, 2010) and increases its acceptability to respondents (Goodman et al, 1997). Moreover, the instrument is designed to allow assessment across a wide age range (3-16 years), which enables longitudinal intra- and intercohort comparison, and it offers multiple informant responses including (depending on the age of the child) child/young person report, parent report and teacher report.

Despite its widespread use as a screening tool (e.g., Children and Young People's Improving Access to Psychological Therapies Outcomes and Evaluation Task and Finish Group, 2011) and outcome measure (Boyer et al, 2014; Hall et al, 2013), the psychometric properties of the SDQ remain under scrutiny. A review of published studies that assessed the psychometric 
properties of the 4-12 year parent and teacher report versions of the SDQ found good levels of internal consistency and satisfactory test-retest reliability and concurrent validity; however, questions remain about the SDQ's construct validity (Stone et al, 2010), with similar findings reported in a systematic review of versions used with pre-echool children (Kersten et al, In Press).

Goodman's (2001) proposed five factor structure has been well supported by studies internationally (eg. Du et al, 2008; Goodman, 2001; Muris et al, 2003; Smedje et al, 1999). However, while Stone et al's (2010) review found evidence in support of this original fivefactor structure, there was acknowledgement that the majority of this evidence was generated using exploratory factor and principle components analysis techniques when the development (i.e, theoretically derived sub-scales) and structure of the SDQ (e.g., limited response options) is more suited to confirmatory factor analysis (Sanne et al, 2009; Stone et al, 2010). Indeed, systematic exploratory and confirmatory testing of the factor structure of the instrument undertaken by Sanne et al (2009) supported a five-factor model but one modified from the original.

Others have suggested that the number of factors needs to be reduced. Dickey and Blumberg (2004), for example, reported a preferred three factor structure composed of the pro-social factor, and internalising and externalising factors in their principle components analysis of the parent report SDQ in America. Koskelainen, et al's (2001) exploratory factor analysis of SDQ responses among 13 - 17 year old Finnish youth, revealed a similar three factor structure. More recently, it has been proposed that the five factor structure may be appropriate as a screening tool in high risk populations and the reduced three factor structure as an outcome measure for epidemiological work (Goodman et al, 2010). 
Alongside the testing of the construct validity of the SDQ there has been consideration of the influence of methodological bias related to the item wording. Goodman (2001) and Dickey and Blumberg (2004) noted that the pro-social factor often included relatively sizeable loadings from positively worded items within other domains, suggesting that this was due to methodological considerations. Similarly Percy et al (2008) reported a lack of unidimensionality in the factor loadings, and particular issues surrounding the reverse worded items. It is proposed by some that the inclusion of a sixth 'method' factor, introduced to account for methodological variance, might facilitate the interpretation of relationships (McCrory et al, 2012; Palmieri et al, 2007; Podsakoff et al, 2003).

Indeed, Van Roy,et al (2008) incorporated such a factor, labelled a 'positive construal factor' in their comparison of alternate models. The positive construal factor was comprised of the 5 positively worded items not included within the pro-social factor, and was correlated with the existing five factors. They reported a better fit of this six factor model for parental and proxy responses. In contrast other researchers have include an uncorrelated method factor, reflecting all ten positively worded items, including those of the pro-social factor ( 5 prosocial, 2, peer, 1 conduct, 2 hyperactivity).

Palmieri and Smith (2007) incorporated this six-factor model in their analysis of SDQ data from custodial grandmothers in the US. Comparing three competing models they concluded that the six-factor model, incorporating an uncorrelated method factor, represented the most appropriate fit to the data. More recently McCrory and Layte (2012) compared four competing models of the parental informant SDQ, the original 5-factor, the 3-factor, a hierarchical factor model (with higher order difficulties factor), and the 6-factor method 
factor model. Using data from the first wave of the Growing Up in Ireland study the authors' confirmatory factor analyses resulted in a preferred 6-factor solution.

Given previously equivocal findings about the factor structure of the SDQ and evidence that this may vary across informant type and across cultures there is a recognised need to continue to build the research base (Dickey et al, 2004; Goodman, 2001; Stone et al, 2010). In particular there is a need to ensure that the psychometric properties of the SDQ are established in countries, such as Scotland, where it is adopted as screening tool and/or population-level indicator. Thus, the present study uses data from parents of children aged 4 years participating in the child cohort of the Growing Up in Scotland study 2006/2007 (ScotCen Social Research, 2013) to explores the factor structure. Based on the findings of previous research, three models were compared: a three-factor, a five-factor and a six-factor (method factor) model (figure 1).

Method

Design and sample: The present study is a secondary data analysis of the child cohort of the 2006/2007 sweep of the Growing Up in Scotland (GUS; ScotCenSocial Research, 2013) study, a longitudinal representative study of children in Scotland. The GUS samples are recruited from a random sample of the Child Benefit Register, covering all local authority areas within Scotland, and consist of a child cohort recruited in 2006/2007 at age 4, and a birth cohort, born between June 2004 and May 2005, recruited at age 10 months (ScotCen Social Research, 2013). The current analysis included parental responses of children from the child cohort at age 4, and is restricted to 2,500 children within the sample for whom parental informant SDQ data was recorded. Analysis of missing data patterns revealed 273 unique 
patterns, this analysis was therefore restricted to a complete case analysis of 1,789 parental responses.

Materials: The parent-informant Strengths and Difficulties Questionnaire (SDQ; Goodman, 1997) was completed by parents of eligible children as part of the GUS study. The SDQ is a 25 item scale, consisting of 5 sub-scales: 4 of which pertain to difficulties (peer problems, emotional difficulties, conduct problems, hyperactivity) which can be summed to create a total difficulties score; and a single strength - pro-social behaviour. The SDQ has a 3 point response scale, with informants asked to indicate their agreements as 'not true' (0), 'somewhat true' (1), or 'certainly true' (2). Higher scores on the difficulties sub-scales (range $0-10)$ and the total difficulties domain (range $0-40$ ) are indicative of greater difficulties, and higher scores on the pro-social sub-scale (range $0-10)$ indicate greater strengths.

Procedure: Data was obtained from the UK data archive, and an effective, analytic sample was extracted which consisted of those cases where parents had completed the SDQ for the cohort child at age 4. Initial statistical analysis was performed in SPSS version 21. Three competing models were specified and estimated using LISREL 8.7 (Jöreskog et al, 2006) and PRELIS 2.7 (Jöreskog et al, 2004), and a maximum likelihood estimation method used. The Chi-squared statistic $(\chi 2)$, normed Chi Square $(\chi 2 / d f)$, Goodness of Fit Index (GFI; Jöreskog et al, 1981), Comparative Fit Index (CFI; Bentler, 1990), Incremental Fit Index (IFI; Bollen, 1989), Root Mean Square Error of Approximation (RMSEA; Steiger, 1990) and 90\% confidence intervals (90\%CI), and the standardised Root Mean Square Residual (SRMR) are presented here as indications of model fit. A non-significant chi square statistic is indicative of a good model fit, however this statistic is sensitive to large sample size (Barrett, 2007). Lower values of the normed chi square are indicative of better fit, with values of less than or 
equal to 5.00 suggesting an adequate fit. Higher values (greater or equal to 0.90) of the GFI, CFI and IFI are indicative of good fit (Bentler, 1981; Jöreskog et al, 1981). RMSEA values lower than 0.07 indicate and acceptable fit, and values less than 0.05 indicate excellent model fit (Steiger, 2007). Similarly lower SRMR values indicate acceptable fit (Hu et al, 1995).

\section{Results}

Model fit indices for the three estimated models are presented in table 1. As can be seen the original 5 factor model was an acceptable fit, while the 3 -factor, pro-socialinternalising-externalising factor structure was a less well-fitting model, with an RMSEA value on the boundary of acceptability. The six factor model, incorporating the uncorrelated method factor of positively worded items, was however the best fitting model in this sample, with an RMSEA indicating an excellent fit for the data.

Comparison of the 5 factor and 6 factor models (table 2) reveal comparable factor loadings within each of the sub-scales, with the exception of those items reflecting the method factor. Generally in the 6 factor solution, items included in the method factor had reduced factor loading on the difficulties factors, than those revealed in the 5 factor model, with one exception. The second conduct problems item 'generally obedient, usually does what adults requests' had a stronger loading on conduct problems in the six factor model than in the five factor model. Variations in the magnitude of the factor loadings between the five and six factor model were, for those items within the peer problems, conduct problems and hyperactivity factors, modest; however for those items within the pro-social domain, factor loadings were greatly reduced in the six factor model, with a greater or comparable loading indicated on the method factor. 
A reliability analysis performed in SPSS, revealed an acceptable reliability for the overall difficulties scale (comprised of 4 subscales), and somewhat lower for the hyperactivity, and pro-social subscales $(0.79,0.68$ and 0.60$)$ respectively. The remaining three sub-scales had less than optimum internal consistency with alpha values below 0.60 , particularly the peer problems sub-scale which had an alpha of $0.34(\alpha=0.49$, conduct problems; $\alpha=0.58$, emotional stability).

\section{Discussion}

The analysis largely supports the original five factor SDQ structure proposed by Goodman (1997), and methodological revision of this structure (McCrory et al, 2012; Palmieri et al, 2007), while the three-factor structure suggested by Dickey and Blumberg (2004) was a poor fit to the data. The findings support the assertion by Palmieri and Smith (2007) that each sub-scale reflects a discrete measure in itself, rather than contributing to more general psychopathology categories. Consistent with previous confirmatory factor analytic studies investigating the presence of a method factor within the SDQ (McCrory et al, 2012; Palmieri et al, 2007; Van Roy et al, 2008) the model fit was improved with the inclusion of a sixth factor, reflecting all 10 positively worded items. This suggests that the SDQ is influenced by methodological bias introduced as a result of measurement (i.e. question phrasing) which might influence the interpretation of the measure (McCrory et al, 2012' Palmieri et al, 2007; Podsakoff et al, 2003). Previous research has shown inconsistencies on the impact of this method factor, Palmieri and Smith (2007) suggesting a marginal influence primarily on the Pro-social factor, evidenced by higher factor loadings on original factors in comparison with the method factor for the majority of items ( 9 out of 10). In contrast McCrory and Layte (2012) reported that three of the five pro-social items loaded 
more highly on the method factor that the original factor. In this case, three pro-social items loaded more highly on the method factor than the pro-social, with the remaining two equivalently loading. Additionally the two positively worded Peer Problems items had considerably reduced loadings on their original factor, and loaded more highly on the method factor. This suggests, that method bias in the SDQ may be more influential than previously reported.

Previous suggestions to rectify the bias in the SDQ have included the omission of the prosocial sub-scale from the measure, however as noted by several researchers (Dickey et al, 2004; Pamieri et al, 2007; Van Roy et al, 2008) there is a case for its continued inclusion. Although introducing a methodological bias within the instrument, the inclusion of positively worded items within the measure is clinically relevant and comparable with alternate measures (Goodman, 1997) and enhances the acceptability of the measure to participants (Dickey et al, 2004). Given the method bias in this analysis is not restricted to the pro-social factor alone, there would appear to be no particular benefit to excluding this sub-scale within the measure.

The reliability analysis revealed lower than expected Cronbach's alphas for all of the subscales, but particularly for the peer problems, conduct problems and emotional stability factors, however, previous research has indicated poor reliability of these sub-scales (Goodman, 2001; Hawes et al, 2004; McCrory et al, 2012; Palmieri et al, 2007). It is worth noting that the SDQ was administered in this case as part of a larger child cohort study, asking parents to respond to an interviewer, which may have influenced their responses. Additionally Stone et al (2010) have suggested that parents may not provide accurate assessments of their children, particularly in relation to peer interactions, thus attenuating the reliability of their responses. 
The analysis supports the emerging research literature indicating the presence of a methodological bias in the Strengths and Difficulties Questionnaire. This analysis of parental informant SDQ data from the Growing Up in Scotland study resulted in a preferred six-factor solution which included a method factor comprised of the 10 positively worded items within the measure. The inclusion of this factor attenuated the factor loadings of several of the positive items on their original factor, and 5 items had higher loading on the method factor than on the original factor. As the SDQ is a widely utilised tool internationally and exists in multiple language versions, suitable for use across a wide age group and from varied informants, more research is warranted to investigate the presence and impact of a method factor, and implications for the interpretation of SDQ scores. 


\section{References}

Barrett, P. (2007). Structural equation modelling Adjusting model fit. Personality and Individual Differences, 42(3), 815 - 824.

Bentler, P.M. (1990). Comparative fit indices in structural models. Psychological Bulletin, $107,238-246$.

Bentler, P.M. (1992). On the fit of models to covariance and methodology to the Bulletin. Psychological Bulletin, 112, 400 - 404.

Bollen, K.A. (1989). A new incremental fit index for general structural models. Sociological Methods and Research, 17, 303 - 316.

Boyer, N., Miller, S., Connolly, P., and McIntosh, E. (2014). Use of the child health utility and strengths and difficulties outcome measures in economic evaluations of school-based interventions: data from a cluster-randomised controlled trial in Northern Ireland. The Lancet, $384, \mathrm{~S} 21$.

Children and Young People's Improving Access to Psychological Therapies Outcomes and Evaluation Task and Finish Group (2011). Children and Young People's Improving Access to Psychological Therapies. Available at: http://www.iapt.nhs.uk/

Dickey, W.C. and Blumberg, S.J. (2004). Revisiting the factor structure of the Strengths and Difficulties Questionnaire: United States, 2001. Journal of the American Academy of Child and Adolescent Psychiatry, 43, $1159-1167$.

Du, Y., Kou, J., and Coghill, D. (2008). The validity, reliability and normative scores of the parent, teacher and self-report versions of the Strengths and Difficulties Questionnaire in China. Child and Adolescent Psychiatry and Mental Healt, $2,8$.

Goodman, R. (1997). The Strengths and Difficulties Questionnaire: A research note. Journal of Child and Adolescent Psychiatry,38, 581 - 586. 
Goodman, R. (2001).Psychometric properties of the Strengths and Difficulties Questionnaire. Journal of the American Academy of Child and Adolescent Psychiatry,40, 1337 - 1345. Hall, C., Moldavsky, M., Baldwin, L., Marriott, M., Newell, K., Taylor, J., Sayal, K. and Hollis, C. (2013). The use of routine outcome measures in two child and adolescent mental health services: a completed audit cycle. BMC Psychiarty, 13, 270.

Hawes, D.J. and Dadds, M.R. (2004). Australian data and psychometric properties of the Strengths and Difficulties Questionnaire. Australian and New Zealand Journal of Psychiatry, $38,644-651$

Hu, L. and Bentler, P.M. (1995). Evaluating model fit. In R.H. Hoyle (Ed.) Structural equation modelling: Concepts, issues, and applications. Thousand Oaks, CA: Sage. Pp. 76 99.

Jöreskog, K. and Sörbom, D. (1981). LISREL V: Analysis of Linear Structural Relationships by the Method of Maximum Likelihood. Chicago: National Educational Resources . Jöreskog, K. and Sörbom, D. (2004). PRELIS 2.70. Chicago: Scientific Software International Inc.

Jöreskog, K. and Sörbom, D. (2006). LISREL 8.70. Chicago: Scientific Software International Inc.

Kersten, P., Czuba, K., McPherson, K., Dudley, M., Elder, H., Tauroa, R., and Vandal, A. (In press). A systematic review of evidence for the psychometric properties of the Strengths and Difficulties Questionnaire. International Journal of Behavioral Development.

Koskelainen, M., Sourander, A. and Vauras, M. (2001). Self-reported strengths and difficulties in a community sample of Finnish adolescents. European Child and Adolescent Psychiatry, 10(3), $180-185$.

Matsuishi, T., Nagano, M., Araki, Y., Tanaka, Y., Iwasaki, M., Yamashita, Y., Nagamitsu, S., Iizuka, C., Ohya, T., Shibuya, K., Hara, M., Matsuda, K., Tsuda, A. and Kakuma, T. (2008). 
Scale properties of the Japanese version of the Strengths and Difficulties Questionnaire (SDQ): A study of infant and school children in community samples. Brain and Development, 30, $410-415$.

McCrory, C. and Layte, R. (2012). Testing competing models of the Strengths and Difficulties Questionnaire's (SDQ's) factor structure for the parent-informant instrument. Personality and Individual Differences, 52, $882-887$.

Muris, P., Meesters, C., and van den Berg, F. (2003). The Strengths and Difficulties Questionnaire (SDQ: Further evidence for its reliability and validity in a community sample of Dutch children and adolescents. European Child and Adolescent Psychiatry, 12, $1-8$. Palmieri, P.A. and Smith, G.C. (2007). Examining the structural validity of the Strengths and Difficulties Questionnaire (SDQ) in a US sample of custodial grandmothers. Psychological Assessment, 19, 189 - 198.

Percy, A., McCrysal, P. and Higgins, K. (2008). Confirmatory factor analysis of the Adolescent Self-report Strengths and Difficulties Questionnaire. European Journal of Psychological Assessment, 24(1), 43 - 48.

Podsakoff, P.M., Mackenzie, S.B., Lee, J.Y. and Podsakoff, N.P. (2003). Common method biases in behavioral research: A critical review of the literature and recommended remedies. Journal of Applied Psychology, 88, 879 - 903.

Schafer, J.L. and Graham, J.W. (2002). Missing Data: our view of the state of the art. Psychological Methods, 7(2), 147 - 177.

ScotCen Social Research (2013). Growing Up in Scotland: Cohort 1, Sweeps 1-6, 2005-2011 [computer file]. 11th Edition. Colchester, Essex: UK Data Archive [distributor], June 2013. SN: 5760, http://dx.doi.org/10.5255/UKDA-SN-5760-4. 
Smedje, H., Broman, J-E., Hetta, J., and von Knorring, A-L. (1999). Psychometric properties of a Swedish version of the 'Strengths and Difficulties Questionnaire'. European Child and Adolescent Psychiatry, 8, $63-70$.

Steiger, J.H. (1990). Structural model evaluation and modification Multivariate Behavioral Research, 25, $212-214$.

Steiger, J.H. (2007). Understanding the limitations of global fit assessment in structural equation modelling. Personality and Individual Differences, 42(3), 893 - 898.

Stone, L.L., Otten, R., Engels, R.C., Vermulst, A.A. and Janssens, J.M. (2010). Psychometric properties of the Strengths and Difficulties Questionnaire for 4-12 year olds: A review. Clinical Child and Family Psychology Review, 13, 254 - 274.

Van Roy, B., Veenstra, M. \& Clench-Aas, J. (2008). Construct validity of te five-factor Strengths and Difficulties Questionnaire (SDQ) in pre-, early and late adolescence. The Journal of Child Psychology and Psychiatry, 49(12), 1304 - 1312. 
Table 1:

Model fit statistics for 3-, 5- and 6-factor models

\begin{tabular}{|c|c|c|c|c|c|c|c|c|c|}
\hline Model & $\chi^{2}$ & df & $\chi^{2} / \mathrm{df}$ & RMSEA & $90 \% \mathrm{CI}$ & CFI & IFI & GFI & SRMR \\
\hline 3 & $1878.30^{* * *}$ & 272 & 6.91 & 0.057 & $0.055-0.060$ & 0.88 & 0.88 & 0.92 & 0.058 \\
\hline 5 & $1558.01 * * *$ & 265 & 5.88 & 0.052 & $0.050-0.055$ & 0.90 & 0.90 & 0.93 & 0.051 \\
\hline 6 & $1175.22 * * *$ & 255 & 4.61 & 0.038 & $0.036-0.040$ & 0.94 & 0.94 & 0.96 & 0.037 \\
\hline
\end{tabular}


Table 2:

Factor loadings for 5-factor model and 6-factor model, method loading in parenthesis 5 -factor 6-factor

\begin{tabular}{|c|c|c|}
\hline \multicolumn{3}{|c|}{ Pro-social } \\
\hline 1 & 0.50 & $0.36(0.36)$ \\
\hline 4 & 0.50 & $0.36(0.36)$ \\
\hline 9 & 0.48 & $0.18(0.47)$ \\
\hline 17 & 0.45 & $0.27(0.40)$ \\
\hline 20 & 0.45 & $0.13(0.51)$ \\
\hline \multicolumn{3}{|c|}{ Peer problems } \\
\hline 6 & 0.44 & 0.46 \\
\hline 11 & -0.27 & $-0.19(0.32)$ \\
\hline 14 & -0.39 & $-0.31(0.36)$ \\
\hline 19 & 0.30 & 0.34 \\
\hline 23 & 0.47 & 0.54 \\
\hline \multicolumn{3}{|c|}{ Conduct } \\
\hline 5 & 0.54 & 0.57 \\
\hline 7 & -0.33 & $-0.47(0.32)$ \\
\hline 12 & 0.50 & 0.52 \\
\hline 18 & 0.39 & 0.41 \\
\hline 22 & 0.21 & 0.21 \\
\hline \multicolumn{3}{|c|}{ Emotion } \\
\hline 3 & 0.37 & 0.38 \\
\hline 8 & 0.52 & 0.52 \\
\hline 13 & 0.46 & 0.46 \\
\hline 16 & 0.48 & 0.48 \\
\hline 24 & 0.56 & 0.56 \\
\hline \multicolumn{3}{|c|}{ Hyperactivity } \\
\hline 2 & 0.70 & 0.72 \\
\hline 10 & 0.62 & 0.63 \\
\hline 15 & 0.67 & 0.67 \\
\hline 21 & -0.39 & $-0.36(0.32)$ \\
\hline 25 & -0.49 & $-0.45(0.32)$ \\
\hline
\end{tabular}


Table 3:

Factor correlations for 5-factor (bottom of diagonal) and 6-factor model (above diagonal)

\begin{tabular}{llllll} 
& Pro-social & Peer & Conduct & Emotional & Hyperactivity \\
\hline Pro-social & & -0.48 & -0.74 & -0.30 & -0.41 \\
Peer & -0.44 & & 0.41 & 0.63 & 0.33 \\
Conduct & -0.54 & 0.44 & & 0.43 & 0.76 \\
Emotional & -0.05 & 0.59 & 0.41 & & 0.33 \\
Hyperactivity & -0.33 & 0.35 & 0.78 & 0.31 & \\
\hline
\end{tabular}




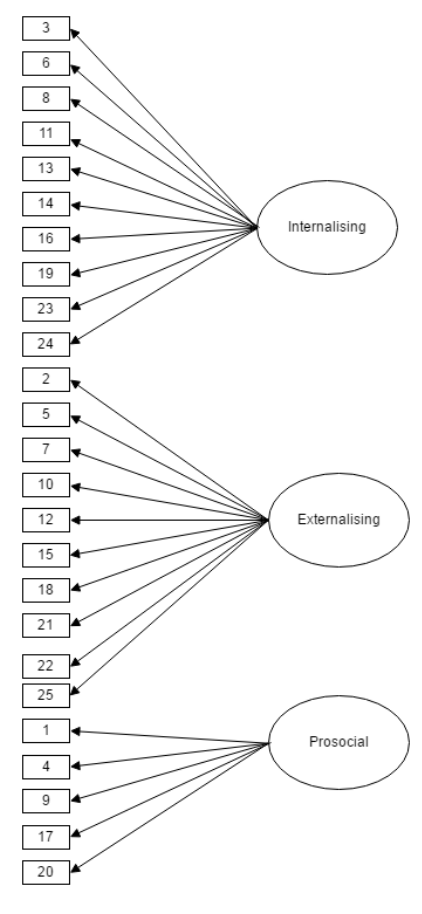

a) 3 Factor

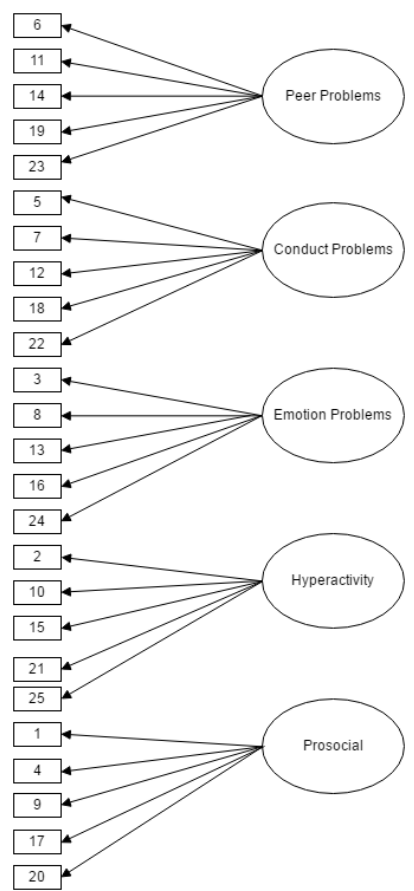

b) 5 Factor

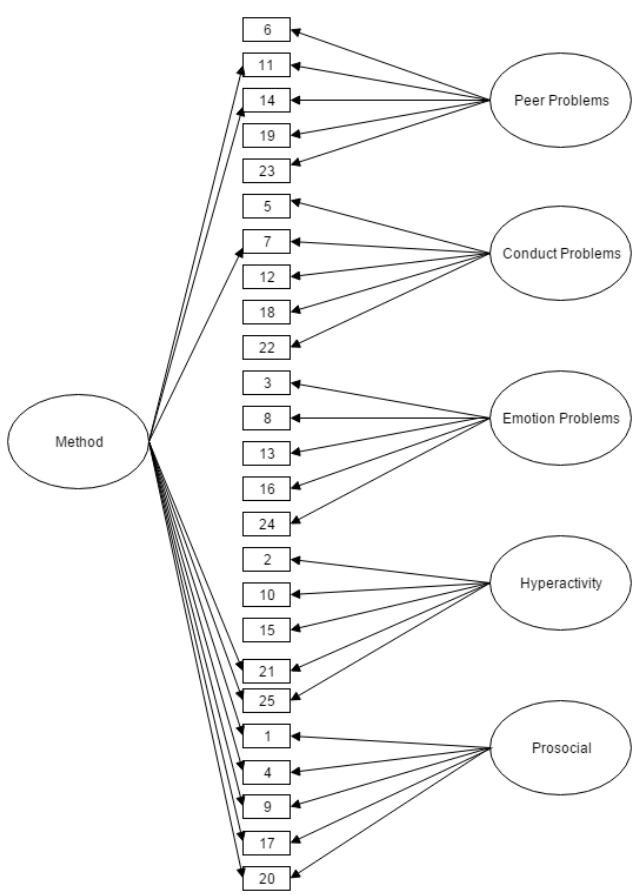

c) 6 Factor (Method factor)

Figure 1:

Conceptual diagrams of model structures 